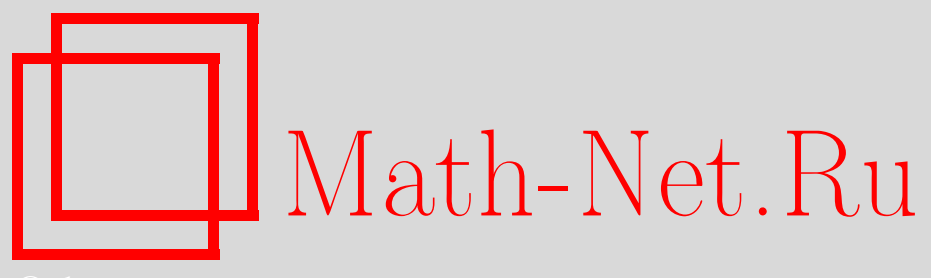

Д. В. Алексеевский, М. В. Белолипецкий, С. Г. Гиндикин, В. Г. Кац, Д. И. Панюшев, Д. А. Тимашев, О. В. Шварцман, А. Г. Элашвили, О. С. Якимова, Эрнест Борисович Винберг (некролог), УМН, 2021, том 76, выпуск 6, 181-192

DOI: https://doi.org/10.4213/rm10030

Использование Общероссийского математического портала Math-Net.Ru подразумевает, что вы прочитали и согласны с пользовательским соглашением http://www . mathnet.ru/rus/agreement

Параметры загрузки:

IP : 54.166 .219 .16

26 апреля 2023 г., 14:34:31

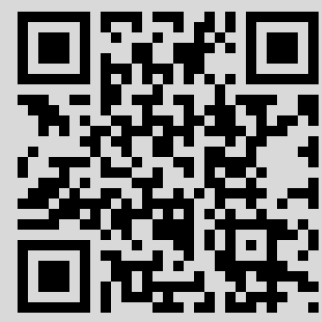




\section{Эрнест Борисович Винберг}

12 мая 2020 г. ушёл из жизни выдающийся математик Эрнест Борисович Винберг.

Эрнест Борисович родился 26 июля 1937 г. в Москве. Его отец Борис Георгиевич Винберг работал инженером-электриком на заводе "Динамо", а мать Вера Евгеньевна Похвальнова преподавала математику и физику, а затем работала инженером-расчётчиком. Во время войны семья Винберга была в эвакуации в Пензенской области, а в 1943 г. вернулась в Москву. С математикой Э. Б. Винберг познакомился и заинтересовался ею очень рано, и уже в средней школе твёрдо решил, что будет математиком. С шесто-

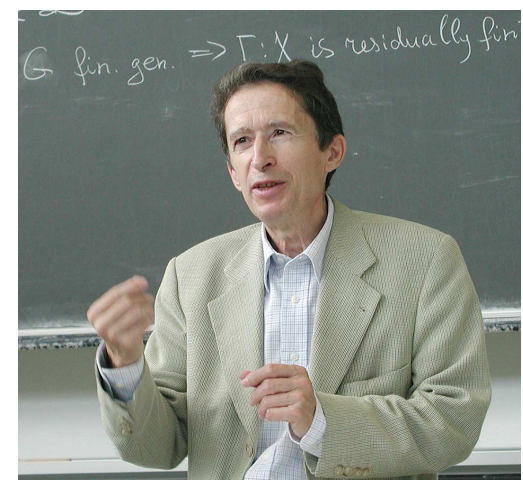
го класса школы он посещал математические кружки в Московском университете на Моховой улице, успешно участвовал в математических олимпиадах и в 1954 г. поступил на механико-математический факультет МГУ.

Научным руководителем Эрнеста Борисовича в студенческие годы и в аспирантуре, куда Э. Б. Винберг поступил после окончания университета в 1959 г., был Евгений Борисович Дынкин. Для Эрнеста Борисовича этот выбор был исключительно важен, и они продолжали поддерживать близкие отношения до смерти Евгения Борисовича. Е.Б. Дынкин был не только выдающимся математиком, но и исключительно оригинальным педагогом. Несомненно, что это обстоятельство сильно повлияло на становление Эрнеста Борисовича как математика и педагога. Для него научные исследования и работа с учениками были одинаково важны.

Главным семинаром для Эрнеста Борисовича был семинар Е. Б. Дынкина по группам Ли, который начался в 1956/57 учебном году как чисто студенческий, но уже на следующий год там появились Ф. А. Березин, Ф. И. Карпелевич, а несколько позже И.И. Пятецкий-Шапиро. Это были состоявшиеся, блестящие молодые математики, в студенческие годы участвовавшие в предыдущей версии семинара Дынкина. Они охотно общались с младшими участниками. Илья Иосифович Пятецкий-Шапиро стал фактически вторым научным руководителем Э. Б. Винберга. Из студентов на семинар постоянно ходили А.А. Кириллов, С. Г. Гиндикин и А. Л. Онищик, поступивший в аспирантуру. K тому времени Дынкин прекратил активную работу в области групп Ли, но сохранил к ней интерес и был очень успешным руководителем семинара. Продолжением семинара Дынкина впоследствии стал известный семинар Винберга-Онищика по группам Ли и теории инвариантов, начатый в 1961/62 учебном году.

В 1961 г. состоялся “колмогоровский” приём новых сотрудников на мехмат МГУ: по инициативе А.Н. Колмогорова и при поддержке ректора МГУ И. Г. Петровского было решено омолодить преподавательский состав, и новый набор был осуществлён в основном из числа однокурсников Э. Б. Винберга. Сам Эрнест Борисович получил

DOI: https://doi.org/10.4213/rm10030 
приглашение от заведующего кафедрой алгебры А.Г. Куроша и, перейдя в заочную аспирантуру, был взят на кафедру ассистентом.

Но ещё за год до этого, будучи аспирантом второго года, Винберг проводил семинарские занятия для студентов первого курса по линейной алгебре. Он вёл их, по свидетельству очевидцев, мастерски и захватывающе интересно. Аура, которую создавал Эрнест Борисович на занятиях, немало способствовала тому, что из студентов тех лет вышло несколько известных математиков. Некоторые из студентов (В. Г. Кац, Б. Н. Кимельфельд, А. Г. Элашвили, а несколько ранее Д. В. Алексеевский и Б. Ю. Вейсфейлер) стали первыми учениками, а потом и соратниками Винберга в научной работе.

В 1962 г. Э. Б. Винберг защитил кандидатскую диссертацию, решив проблему об однородных выпуклых конусах и областях Зигеля, поставленную Пятецким-Шапиро (подробнее об этом см. ниже). В 1965 г. по предложению Куроша Эрнест Борисович прочёл свой первый (не считая спецкурсов) курс лекций по линейной алгебре и геометрии и в том же году получил должность доцента.

В начале 1970-х годов в академической карьере (но не в научном творчестве!) Эрнеста Борисовича наступил трудный период. Винберг был в числе подписавших "письмо девяноста девяти" в защиту математика А.С. Есенина-Вольпина, насильственно помещённого в психиатрическую больницу по политическим мотивам, после чего последовала реакция. Для Эрнеста Борисовича наступил длительный перерыв в чтении лекций и возникли препятствия к руководству аспирантами на мехмате, а докторскую диссертацию ему пришлось защищать дважды - в 1971 и 1984 гг. ${ }^{1}$ (обе работы относились к гиперболическим группам отражений - см. ниже о работах Винберга в этой области).

Положение изменилось с началом перестройки. В 1990 г. Эрнест Борисович стал профессором кафедры алгебры мехмата МГУ и работал на кафедре, читая лекции и спецкурсы, ведя семинары и руководя научной работой своих учеников, до конца дней.

Научное творчество Э. Б. Винберга исключительно широко и разнообразно, причём интересы Эрнеста Борисовича оставались многообразными на протяжении всей его жизни. Это легко понять из описания его основных достижений в математике, приведённого ниже и далеко не исчерпывающего всех областей, которые он обогатил новыми идеями, понятиями и результатами.

До самых последних дней Эрнест Борисович успешно работал во многих областях и творчески общался со своими учениками и коллегами. Он неоднократно помогал советами как математикам, так и людям иных специальностей, которые обращались к нему с математическими вопросами и всегда получали квалифицированный ответ по существу.

Говоря о влиянии печатных работ Э. Б. Винберга, хочется отметить, что все они представляют собой тексты, которые можно понять и из которых можно многому научитъся. В них присутствует неуловимое умение доступного и понятного изложения. Разнообразные примеры, интересные замечания и открытые проблемы, упоминаемые в работах Винберга, дают понять заинтересованному читателю, что написанное представляет только вершину айсберга, и подсказывают темы для дальнейших раздумий и собственных исследований. Эти качества, в частности, проявились в серии энциклопедических обзоров [22]-[27] в "Итогах науки и техники" ВИНИТИ, написанных Э. Б. Винбергом с соавторами в конце 1980-х годов. Стоит также заметить, что русский язык и стиль статей и книг Эрнеста Борисовича был безукоризненным.

Ведя активную исследовательскую работу и публикуя собственные результаты (около 150 научных работ в списке публикаций на www.mathnet.ru), Э.Б. Винберг

${ }^{1}$ См. статью С. П. Новикова "Защита Винберга (13-летняя эпопея в московской математике 70-80-х годов)" на его странице в Math-Net.Ru. - Прим. гл. ред. 
отдавал много сил редакционной деятельности, состоя в редколлегиях ряда ведущих математических журналов: “Функциональный анализ и его приложения" (с 2005 г.), "Труды Московского математического общества" (с 1999 г.), "Journal of Lie Theory" (с 1994 г.). Эрнест Борисович был бессменным главным редактором журнала "Transformation Groups" с момента его основания (1996 г.).

Эрнест Борисович Винберг был не только выдающимся математиком, но и замечательным педагогом, Учителем. У него было много учеников. Годы ученичества под руководством Винберга затем перерастали в научное сотрудничество, которое продолжалось до конца его дней. За эти годы легко было убедиться в исключительности таланта Эрнеста Борисовича как педагога и исследователя. Этот талант позволил ему воспитать плеяду первоклассных математиков и обнаруживать математические факты и пути их доказательства, которые никто кроме него обнаружить бы не смог. Как педагога его отличала интуиция, терпение и исключительная ясность изложения проблем, которые он ставил своим ученикам. Случалось, что эти проблемы были далеки от непосредственной научной работы Әрнеста Борисовича, но они оказывались толчком к развитию новых направлений.

Почти все ученики Э. Б. Винберга выросли на семинаре по группам Ли на мехмате МГУ, которым он руководил с 1961 г. совместно с А.Л. Онищиком, а впоследствии и с некоторыми своими учениками. Семинар Винберга-Онищика на протяжении более полувека был значительным событием московской математической жизни. Записки семинара легли в основу книги [21] (1988 г.), которую отличает нестандартный и оригинальный подход к изложению теории групп и алгебр Ли, делающий её уникальным источником как для специалистов, так и для неофитов, приступающих к изучению этой области.

В начале 1990-х годов Эрнест Борисович принимал деятельное участие в организации Независимого Московского университета и читал там базовые, но весьма нестандартные и глубокие курсы алгебры. Из записок этих лекций, изданных в НМУ в 1995 г., впоследствии вырос его "Курс алгебры" [30], выдержавший много переизданий и ставший классическим университетским учебником. В последние пятнадцать лет жизни Э. Б. Винберг был главным редактором журнала "Математическое просвещение".

Эрнест Борисович был научным руководителем серии летних и зимних школ "Алгебры Ли, алгебраические группы и теория инвариантов", проводившихся попеременно в Самарской области и в Москве во второй декаде XXI в., первая из которых состоялась под Самарой в июне 2009 г., а последняя на данный момент, девятая по счёту, - в Самаре в августе 2021 г. Э.Б. Винберг был неизменным председателем программного комитета и постоянным лектором этих школ. Он придавал им большое значение в деле распространения современных математических знаний, стараясь в своих лекционных курсах (2009, 2011, 2014, 2017 гг.) в доступной форме, с присущим ему мастерством познакомить молодых слушателей с новейшими результатами в тех областях, которыми он занимался.

В 1997 г. Э. Б. Винберг получил премию Александра фон Гумбольдта, на которую его выдвинул университет Билефельда. Это положило начало более чем двадцатилетнему периоду сотрудничества Винберга с математиками из Билефельда (Г. Абельс, Й. Меннике, Х. Хеллинг) и регулярным летним визитам в Германию. Для Эрнеста Борисовича это составляло важную часть жизни, поскольку там, вдали от московской суеты, он мог полностью отдаться научному творчеству. Вместе с тем, будучи искренним патриотом, Эрнест Борисович никогда не рассматривал возможность эмиграции, не мысля свою жизнь вне Родины.

Он был необыкновенно цельным человеком, очень интеллигентным и доброжелательным, но своими принципами не поступался никогда. С его уходом математическая жизнь уже не будет прежней. 
Перечислим самые яркие достижения Эрнеста Борисовича Винберга, которые навсегда останутся в математике. Подробный рассказ о научной деятельности Винберга и его вкладе в математику можно найти в расширенной версии данной статьи на arXiv.org (см. arXiv: 2108.02544).

Однородные комплексные области и выпуклые конусы. В 1959 г. И. И. Пятецкий-Шапиро сделал сенсационное открытие, построив пример несимметрической однородной ограниченной области в $\mathbb{C}^{4}$. Тем самым была решена одна из старых проблем Э. Картана. Одновременно возник проект, целью которого была классификация однородных ограниченных областей в $\mathbb{C}^{n}$. Участвовать в проекте Пятецкий-Шапиро пригласил Э.Б. Винберга и С.Г. Гиндикина. Поставленные им задачи и его точки зрения решающим образом повлияли на становление Винберга как математика.

Главную роль в классификации сыграли открытые Пятецким-Шапиро области Зигеля второго рода. Такая область строится по выпуклому вещественному конусу и векторнозначной эрмитовой форме, положительной относительно рассматриваемого конуса. Перед Эрнестом Борисовичем была поставлена задача понять природу выпуклых однородных конусов, которые появляются в областях Зигеля с классической группой автоморфизмов.

Эти конусы являются выпуклыми, линейно однородными, не содержат прямых и являются самосопряжёнными для некоторых положительных билинейных форм. Винберг увидел, что классификация таких конусов эквивалентна классификации компактных йордановых алгебр, которая была известна. Несколько раньше эту связь рассматривал М. Кёхер. Винберг ссылался на некоторые результаты Кёхера, но о его основной теореме он узнал только после сдачи заметки в печать. Сегодня этот результат называется теоремой Кёхера-Винберга.

Эрнест Борисович показал, что существуют четыре серии конусов, связанных с йордановыми алгебрами, которые в точности отвечают четырём сериям классических областей. Есть ещё особый конус, связанный с октавными матрицами третьего порядка. Он отвечает реализации особой 27-мерной комплексной симметрической области как области Зигеля.

Работа Винберга о конусах [1] (1960 г.) содержала ещё один важнейший результат: пример выпуклого линейно однородного, но не самосопряжённого пятимерного конуса (в меньших размерностях таких нет), что автоматически приводит к примеру несимметрической аффинно однородной области Зигеля в размерности 5. Параллельно Винберг построил континуум неэквивалентных выпуклых линейно однородных конусов в размерности 11 .

Какое-то время казалось, что понятие областей Зигеля нуждается в расширении, чтобы охватить все комплексные однородные области. Однако в 1963 г. Э. Б. Винберг, С. Г. Гиндикин и И. И. Пятецкий-Шапиро опубликовали фундаментальный результат теории.

Теорема [3]. Всякая комплексная однородная ограниченная область биголоморфно эквивалентна афбинно однородной области Зигеля второго рода.

При доказательстве этого результата стало ясно, что решающую роль играет наличие на упомянутых областях структуры однородных кэлеровых многообразий. На этом основании Э.Б. Винберг и С.Г. Гиндикин рассмотрели случай произвольных однородных кэлеровых многообразий. Возникла гипотеза, что произвольное однородное кэлерово многообразие допускает однородное расслоение с комплексной ограниченной однородной областью в качестве базы и прямыми произведениями локально плоских и односвязных компактных кэлеровых однородных многообразий в качестве слоев.

В 1967 г. Винберг и Гиндикин доказали гипотезу для центрального случая разрешимых групп автоморфизмов [5]. Окончательно гипотеза была доказана в 1995 г. 
Теория однородных выпуклых конусов и областей составила содержание кандидатской диссертации Э.Б. Винберга, см. [2] (1963 г.). За эти результаты Винберг получил премию Московского математического общества (1963 г.).

Теория однородных выпуклых конусов не только занимает особое место в творчестве Э. Б. Винберга, но и находит удивительные применения далеко за пределами этой области математики. Оказалось, что эта теория допускает интерпретацию в рамках информационной геометрии Ченцова-Амари, тесно связана с геометрической термодинамикой, в частности с термодинамикой Сурьо на группах Ли и её обобщениями, широко используется в современной математике и теоретической физике, например для унификации и обобщения магического квадрата Фрейденталя и магической звезды Мукаи и при изучении чёрных дыр в $N=2$ супергравитации.

Группы отражений в пространствах Лобачевского. В середине 1960-х годов Эрнест Борисович начал заниматься дискретными группами, порождёнными отражениями в гиперплоскостях в пространстве Лобачевского $\Lambda^{n}$ (гиперболическими калейдоскопами, как он любил их называть). В 1967 г. в "Математическом сборнике" вышла его статья [4]. Эта работа состоит из трёх частей. В первой, геометрической, части строится систематическая теория гиперболических групп отражений в терминах матриц Грама фундаментальных многогранников, обобщающая классическую евклидову теорию Кокстера. Во второй, алгебраической, части получен критерий арифметичности гиперболической группы отражений в терминах матрицы Грама её фундаментального многогранника. Здесь же был определён класс квазиарифметических подгрупп, которые привлекают значительный интерес в последние годы. В третьей, заключительной, части работы Винберг применяет критерий арифметичности для построения и анализа конкретных примеров арифметических и неарифметических групп отражений в $\Lambda^{n}$. В частности, здесь естественным образом получены известные ранее примеры В.С. Макарова. На протяжении более пятидесяти лет фундаментальная и яркая статья Винберга [4] является одной из основных ссылок в данной области.

В 1972 г. в "Математическом сборнике" вышла работа Винберга [7]. В ней описывается получивший впоследствии широкую известность алгоритм Винберга для построения арифметических групп, порождённых отражениями. С тех пор этот алгоритм стал одним из главных инструментов любого математика, работающего с гиперболическими группами отражений. Примеры, полученные с его помощью в [7] и в последующих работах, нашли применение в работах Дж. Конвея и Р. Борчердса по гипотезе "moonshine" (как говорил Конвей, "without Vinberg groups there would be no monstrous moonshine").

В начале 1980-х годов Эрнест Борисович доказал глубокий и неожиданный результат об отсутствии решёток, порождённых отражениями, в пространствах $\Lambda^{n}$ большой размерности.

Tеорема [17], [18]. В пространствах Лобачевского размерности $n \geqslant 30$ не существует дискретных групп отражений с ограниченным фундаментальным многогранником и арифметических дискретных групп отражений.

Впоследствии, развивая некоторые идеи Винберга, М.Н. Прохоров доказал, что для $n \geqslant 996$ в $\Lambda^{n}$ не существует также и дискретных групп отражений с неограниченным фундаментальным многогранником конечного объёма. Группы отражений в пространствах Лобачевского и теоремы конечности были представлены Винбергом в его докладе на Международном конгрессе математиков в Варшаве [19] (1984 г.).

Что происходит в тех размерностях, в которых существуют решётки и арифметические группы, порождённые отражениями? В размерности 2 полная классификация рефлективных решёток принадлежит Ф. Клейну и А. Пуанкаре. В размерности 3 классификация была получена Е. М. Андреевым, когда он учился в аспирантуре 
под руководством Винберга (1970 г.). Работы Андреева впоследствии легли в основу теории геометризации трёхмерных многообразий У.П. Тёрстона. Систематическое изучение вопросов классификации арифметических групп отражений было начато в 1980-е годы в работах В. В. Никулина и Э. Б. Винберга и затем продолжено другими исследователями.

Э. Б. Винберг продолжал заниматься группами отражений в пространствах Лобачевского на протяжении всей математической карьеры, открывая всё новые горизонты для дальнейших исследований.

Алгебраическая теория инвариантов. Задачи теории инвариантов в широком смысле привлекали Эрнеста Борисовича со студенческих лет. В совместной работе Э.Б. Винберга с учениками [6] (1967 г.) исследуется вопрос о тривиальности стационарной подалгебры общего положения (т. е. алгебры Ли стабилизатора вектора общего положения) для линейного представления комплексной полупростой группы Ли $G$. Свойства нетривиальности стационарных подалгебр и стационарных подгрупп (стабилизаторов) векторов общего положения коррелируют с другими "хорошими" теоретико-инвариантными свойствами линейных представлений: алгебраической независимостью базисных инвариантных многочленов (порождающих алгебру инвариантов представления) и конечностью числа орбит в любом многообразии уровня инвариантных многочленов (где инварианты принимают заданные значения). Таких "хороших" представлений сравнительно немного, но они наиболее интересны с точки зрения теории инвариантов и её многочисленных приложений.

В работе [11] (1976 г.) Э. Б. Винберг, В. Г. Кац и В. Л. Попов получили список всех неприводимых представлений простых комплексных групп Ли со свободной алгеброй инвариантов, который совпал с ранее найденными списками линейных представлений в указанном классе, имеющих нетривиальный стабилизатор общего положения или конечное число орбит в любом многообразии уровня инвариантов.

Вопросы о причинах "хорошего" теоретико-инвариантного поведения линейных представлений и естественном источнике таких представлений давно интересовали исследователей. В своей фундаментальной работе 1963 г. Б. Костант построил теорию инвариантов присоединённого представления полупростой комплексной группы Ли $G$ в её касательной алгебре Ли g. В частности, обнаружилось, что присоединённое представление обладает указанными выше "хорошими" свойствами. Через десяток лет после работы Костанта Э.Б. Винберг далеко развил и обобщил его результаты, создав новое направление в теории инвариантов - теорию тета-групп.

Пусть полупростая алгебра Ли $\mathfrak{g}$ снабжена периодической градуировкой по модулю $m$ :

$$
\mathfrak{g}=\mathfrak{g}_{0} \oplus \mathfrak{g}_{1} \oplus \cdots \oplus \mathfrak{g}_{m-1} .
$$

Эта градуировка задаётся периодическим автоморфизмом $\theta$ алгебры $\mathfrak{g}$. Связная подгруппа Ли $G_{0} \subset G$, соответствующая подалгебре Ли $\mathfrak{g}_{0} \subset \mathfrak{g}$, действует в пространстве $\mathfrak{g}_{1}$ путём ограничения присоединённого представления группы $G$. Образ группы $G_{0}$ при этом представлении называется $\theta$-группой градуированной алгебры $\mathfrak{g}$.

В $1973-1975$ гг. Э. Б. Винберг доказал, что алгебра $G_{0}$-инвариантных многочленов на пространстве $\mathfrak{g}_{1}$ свободна, и описал замкнутые $G_{0}$-орбиты в пространстве $\mathfrak{g}_{1}$ [10], [12]. В то же время Эрнестом Борисовичем был предложен метод носителей [9], [16] для классификации нильпотентных $G_{0}$-орбит в пространстве $\mathfrak{g}_{1}$. Вместе с описанием замкнутых (полупростых) орбит и градуированной версией разложения Жордана в полупростой алгебре Ли, этот результат позволяет в принципе описать все орбиты группы $G_{0}$ в пространстве $\mathfrak{g}_{1}$, что является конечной целью теории инвариантов для данного линейного представления. Испытательным полигоном для этого метода послужила полученная Э. Б. Винбергом и А. Г. Элашвили классификация тривекторов девятимерного пространства [14] (1978 г.), что продолжило классические исследования В. Райхеля, Я. А. Схоутена и Г. Б. Гуревича. 
Теория тета-групп оказалась чрезвычайно полезной не только в теории инвариантов, теории представлений и теории алгебраических групп, но и в теории чисел, и в алгебраической геометрии, и теперь заслуженно называется теорией тета-групп Винберга.

Тета-группы почти исчерпывают список "хороших" неприводимых представлений простых групп Ли, о котором говорилось выше. Наиболее интересный из оставшихся случаев - спинорное представление группы $\operatorname{Spin}_{13}$ - был полностью исследован в работе Э. Б. Винберга (Е. Viniberghi) и В. Г. Каца (V. Gatti) [13] (1978 г.). По совету Ж.-К. Рота авторы взяли себе псевдонимы: Katze - "кошка" по-немецки, a gatta по-итальянски; Viniberghi - итальянизированное "Винберг". В то время публикация статьи в соавторстве с Кацем, к тому времени эмигрировавшим в США, могла навредить Винбергу.

Алгебраические группы преобразований. Алгебраическая теория инвариантов является частью более общей науки - теории алгебраических групп преобразований, изучающей действия алгебраических групп на алгебраических многообразиях и их влияние на геометрию многообразий и теорию представлений. Эрнест Борисович начал работать в этой области в 1970-х годах.

В совместной работе Э. Б. Винберга с Б. Н. Кимельфельдом [15] (1978 г.) получен следующий результат.

Теорема. Действие борелевской подгруппы В полупростой комплексной группы Ли $G$ на однородном пространстве $G / H$ имеет открытую плотную орбиту тогда и толъко тогда, когда представление группы $G$ в пространстве сечений любого однородного линейного расслоения $\mathcal{L} \rightarrow G / H$ имеет простой спектр.

Эта теорема связывает эквивариантную геометрию с гармоническим анализом на однородных пространствах, выделяя ключевое свойство наличия открытой $B$-орбиты. Однородные пространства или, более общо, алгебраические многообразия, снабжённые действием редуктивной группы $G$, для которого борелевская подгруппа $B \subset G$ имеет открытую орбиту, получили название сферических. Теорема Винберга-Кимельфельда - один из краеугольных камней теории сферических многообразий, которая интенсивно развивается по сей день.

Вклад Э. Б. Винберга в теорию сферических многообразий включает в себя также его совместную работу с В.Л. Поповым [8] (1972 г.), где для изучения одного класса сферических многообразий (которые тогда ещё так не назывались) была впервые развита комбинаторно-геометрическая техника полиэдральных конусов и решёток, ставшая впоследствии одним из основных инструментов изучения сферических многообразий и их частного случая - торических многообразий, теория которых только зарождалась в те годы (начало 1970-х) в работах М. Демазюра и др.

$\mathrm{K}$ теории сферических многообразий относится и полученное Винбергом в работе [29] (1995 г.) полное описание редуктивных алгебраических полугрупп (т. е. алгебраических многообразий с ассоциативной операцией умножения, единицей и редуктивной группой обратимых элементов) с детальным исследованием их алгебраических и геометрических свойств.

Осмысление важной роли действия борелевской подгруппы привело Эрнеста Борисовича к введению в работе [20] (1986 г.) понятия сложности действия редуктивной алгебраической группы $G$ на неприводимом алгебраическом многообразии $X$ : под сложностью действия понимается коразмерность орбит общего положения борелевской подгруппы $B \subset G$ в $X$. Ключевой результат состоит в следующем.

Теорема [20]. Если $Y$ - произвольное неприводимое B-инвариантное подмногообразие в $X$, то коразмерность $B$-орбит общего положения в $Y$ не превосходит сложности действия $G$ на $X$. 
Таким образом, наиболее "богатое" непрерывное семейство борелевских орбит состоит из орбит общего положения. Следует отметить, что, хотя утверждение касается действия группы $B$, наличие действия объемлющей редуктивной группы $G$ существенно: без этого условия утверждение перестаёт быть верным. Для действий сложности 0 (т. е. сферических) получается такое важное утверждение.

СлЕДСтвиЕ. Если группа $B$ действует на G-многообразии $X$ с открытой плотной орбитой, то она имеет лищь конечное число орбит в $X$.

Последнее утверждение независимо доказал М. Брион. Это замечательное свойство сферических многообразий по сути даёт их клеточные разбиения, аналогичные разбиениям многообразий флагов на клетки Шуберта, и имеет многочисленные геометрические, топологические и теоретико-представленческие следствия.

В целом сложность оказалась тем инвариантом действия редуктивной группы, который отделяет "ручные" (поддающиеся исчерпывающему описанию) действия от "диких" (полная теория которых вряд ли возможна). К настоящему времени получена полная классификация сферических однородных пространств и комбинаторногеометрическое описание их эквивариантных открытых вложений - сферических многообразий. Случай сложности 1 тоже до некоторой степени поддаётся описанию, в то время как насчёт действий сложности $>1$ сложилось понимание, что полное их описание в каком-либо разумном смысле вряд ли возможно.

Пуассонова алгебра и симплектическая геометрия. Первое обращение Эрнеста Борисовича к изучению пуассоновой структуры, связанной с коприсоединённым представлением группы Ли, и эквивариантной симплектической геометрии произошло в статье [28] (1990 г.). В этой работе делается попытка квантования коммутативных (по Пуассону) подалгебр в симметрической алгебре $\mathcal{S}(\mathfrak{g})$ полупростой алгебры Ли $\mathfrak{g}$, получаемых методом сдвига аргумента. Это сделано для элементов степени 2 с помощью явной конструкции, которая потом использовалась другими авторами и для более общих целей.

Квантование "больших" коммутативных подалгебр в $\mathcal{S}(\mathfrak{g})$ (например, имеющих максимальную возможную степень трансцендентности, как подалгебры, получаемые сдвигом аргумента) приводит к таким же коммутативным подалгебрам в универсальной обёртывающей алгебре $\mathcal{U}(\mathfrak{g})$, играющим первостепенную роль в теории представлений. На эту роль обратили внимание ещё И. М. Гельфанд и М. Л. Цетлин в известных работах 1950 г., посвящённых построению канонических базисов в конечномерных представлениях алгебр Ли $\mathfrak{s l}_{n}$ и $\mathfrak{s o}_{n}$. В работе [28] показано, что в случае $\mathfrak{g}=\mathfrak{s l}_{n}$ деквантование подалгебры Гельфанда-Цетлина является пределом подалгебр, получаемых сдвигом аргумента. В дальнейшем эта тематика заняла значительное место в деятельности как самого Эрнеста Борисовича, так и его учеников и последователей.

В совместной работе [31] (1999 г.) Д.Н.Ахиезер и Э. Б. Винберг перенесли понятие слабо симметрического пространства, введённое А. Сельбергом для римановых многообразий в известной статье 1956 г. о формуле следа, на аффинные однородные многообразия редуктивных алгебраических групп. Основной результат работы состоит в том, что аффинное однородное многообразие слабо симметрично тогда и только тогда, когда оно сферично. В частности, сферические аффинные комплексные однородные многообразия суть комплексификации компактных слабо симметрических римановых многообразий Сельберга.

Слабо симметрические римановы однородные многообразия $X$ являются коммутативными, т. е. алгебра инвариантных дифференциальных операторов на $X$ коммутативна; это показал ещё Сельберг. Для риманова однородного многообразия $X=G / K$ коммутативность - это одно из эквивалентных определяющих свойств 
пары Гельфанда $(G, K)$ в классическом её понимании. Как показано в [31], риманово многообразие $X=G / K$ с редуктивной транзитивной группой изометрий $G$ коммутативно тогда и только тогда, когда оно слабо симметрично, т. е. его комплексификация сферична. Таким образом, была обнаружена связь между свойством сферичности и парами Гельфанда, что позволило перенести изучение коммутативных пространств и пар Гельфанда на алгебро-геометрическую почву.

Две основополагающие статьи Э. Б. Винберга [32], [33] (2001 и 2003 гг.) посвящены коммутативным однородным пространствам вещественных групп Ли (не обязательно редуктивных). Эрнест Борисович получил ряд структурных результатов о коммутативных пространствах, которые позволили ему классифицировать нильпотентные коммутативные пространства (т. е. такие, для которых $G=K \ltimes N$, а группа $N$ унипотентна). В дальнейшем эти результаты привели к полной классификации пар Гельфанда в диссертации О. С. Якимовой (2005 г.), выполненной под руководством Винберга.

Если однородное пространство $X=G / K$ коммутативно, то действие $G$ на $T^{*} X \kappa o$ изотропно, т. е. касательные пространства к орбитам общего положения коизотропны (в смысле стандартной симплектической структуры кокасательного расслоения). На момент написания статьи [32] обратная импликация была хорошо известна в случае редуктивной группы $G$. Э. Б. Винберг показал, что она верна и для нильпотентных однородных пространств. Позднее Л. Г. Рыбников доказал эквивалентность в общем случае (2003 г.).

Коизотропные действия, а также вопросы, связанные с корангом симплектической формы на орбитах общего положения, относятся к предмету эквивариантной симплектической геометрии. В качестве приложений здесь получаются утверждения об интегрируемых гамильтоновых системах. В случае комплексной редуктивной группы $G$, действующей на алгебраическом многообразии $X$, коранг естественного действия $G$ на кокасательном расслоении $T^{*} X$ равен удвоенной сложности действия $G$ на $X$. В частности, действие $G$ на $T^{*} X$ коизотропно тогда и только тогда, когда действие $G$ на $X$ сферично. В работе [32] дано новое доказательство этого факта, основанное на применении орисфер (т. е. орбит максимальных унипотентных подгрупп группы $G)$.

“Трансцендентная теория инвариантов" и автоморфные формы. В 2009 г. Э. Б. Винберг возвращается к однородным симметрическим областям в связи с задачей о свободных алгебрах автоморфных форм на эрмитовых симметрических пространствах некомпактного типа.

Пусть $X$ - такое пространство, $G$ - группа аналитических автоморфизмов пространства $X$, а $\Gamma \subset G$ - дискретная подгруппа с факторпространством конечного объёма. Алгебра $A(\Gamma)$ Г-автоморфных форм (относительно канонического фактора автоморфности) является неотрицательно градуированной алгеброй конечного типа. Пару $(X, \Gamma)$ назовём парой Шевалле, если алгебра $A(\Gamma)$ свободна.

Задача состоит в том, чтобы перечислить все такие пары (с точностью до естественной эквивалентности). Постановка задачи мотивирована классической теоремой Шевалле о свободных алгебрах инвариантов конечных линейных групп.

Для того чтобы пара $(X, \Gamma)$ была парой Шевалле, необходимо, чтобы группа $\Gamma$ порождалась комплексными отражениями. Это условие оставляет для пространства $X$ только три возможности: комплексный шар, полидиск или область $D_{n}$ четвёртого типа по классификации Э. Картана ( $D_{2}$ - это произведение двух верхних полуплоскостей, $D_{3}$ - трёхмерная верхняя полуплоскость Зигеля рода 2). Резонансный пример пары Шевалле $\left(D_{3}, \mathrm{Sp}_{4}(\mathbb{Z})\right)$ был найден Игузой почти шестьдесят лет тому назад, и на протяжении многих лет спорадические примеры пар Шевалле в размерностях, не больших 4, встречались в статьях разных авторов. 
Прорыв произошёл в 2010 г., когда появилась первая из двух работ [34], [36] Э.Б. Винберга, в которых впервые были построены примеры пар Шевалле вида $\left(D_{n}, \Gamma\right)$ для всех размерностей $5 \leqslant n \leqslant 10$. А в 2017 г., в совместной работе [35] Э. Б. Винберга и О.В. Шварцмана, было показано, что пары такого вида могут существовать только при $n \leqslant 10$. Приступить к классификации дискретных групп, действующих в областях $D_{n}$, со свободной алгеброй автоморфных форм Эрнест Борисович уже не успел, и те, кто доведут дело до конца, будут многим ему обязаны.

\section{Д.В. Алексеевский, М.В. Белолипецкий, С.Г. Гиндикин, В.Г. Кач, Д. И. Панюшев, Д. А. Тимашев, О. В. Швариман, А.Г. Элашвили, О.С. Якимова}

\section{Список цитированных работ Э.Б. Винберга}

[1] Э. Б. Винберг, "Однородные конусы”, Докл. АН СССР, 133:1 (1960), 9-12; англ. пер.: 亡̀. B. Vinberg, "Homogeneous cones", Soviet Math. Dokl., 1 (1960), 787-790.

[2] Э. Б. Винберг, "Теория однородных выпуклых конусов", Тр. ММО, 12, ГИФМЛ, M., 1963, 303-358; англ. пер.: Е. B. Vinberg, "The theory of convex homogeneous cones", Trans. Moscow Math. Soc., 12 (1963), 340-403.

[3] Э.Б. Винберг, С.Г. Гиндикин, И.И. Пятецкий-Шапиро, "О классификации и канонической реализации комплексных однородных ограниченных областей”, Тр. ММО, 12, ГИФМЛ, М., 1963, 359-388; англ. пер.: Ѐ.В. Vinberg, I. I. Piatetski-Shapiro, "Classification and canonical realization of complex bounded homogeneous domains", Trans. Moscow Math. Soc., 12 (1963), 404-437.

[4] Э.Б. Винберг, "Дискретные группы, порожденные отражениями, в пространствах Лобачевского", Матем. сб., 72(114):3 (1967), 471-488; англ. пер.: È. B. Vinberg, "Discrete groups generated by reflections in Lobachevskiu spaces", Math. USSR-Sb., 1:3 (1967), 429-444.

[5] Э.Б. Винберг, С.Г. Гиндикин, "Кэлеровы многообразия, допускающие транзитивную разрешимую группу автоморфизмов", Матем. сб., 74(116):3 (1967), 357-377; англ. пер.: Ѐ. B. Vinberg, S. G. Gindikin, "Kählerian manifolds admitting a transitive solvable automorphism group", Math. USSR-Sb., 3:3 (1967), 333-351.

[6] Е. М. Андреев, Э.Б. Винберг, А.Г. Элашвили, "Орбиты наибольшей размерности полупростых линейных групп Ли", Функи. анализ и его прил., 1:4 (1967), 3-7; англ. пер.: E. M. Andreev, É. B. Vinberg, A. G. Élashvili, "Orbits of greatest dimension in semi-simple linear Lie groups", Funct. Anal. Appl., 1:4 (1967), 257-261.

[7] Э. Б. Винберг, "О группах единиц некоторых квадратичных форм", Матем. сб., 87(129):1 (1972), 18-36; англ. пер.: Ё. В. Vinberg, "On groups of unit elements of certain quadratic forms", Math. USSR-Sb., 16:1 (1972), 17-35.

[8] Э. Б. Винберг, В. Л. Попов, "Об одном классе квазиоднородных аффинных многообразий”, Изв. АН СССР. Сер. матем., 36:4 (1972), 749-764; англ. пер.: È. B. Vinberg, V.L. Popov, "On a class of quasihomogeneous affine varieties", Math. USSR-Izv., 6:4 (1972), 743-758.

[9] Э. Б. Винберг, "О классификации нильпотентных элементов градуированных алгебр Ли”, Докл. АН СССР, 225:4 (1975), 745-748; англ. пер.: Е. В. Vinberg, "On the classification of the nilpotent elements of graded Lie algebras", Soviet Math. Dokl., 16(1975) (1976), 1517-1520.

[10] Э.В. Винберг, "О линейных группах, связанных с периодическими автоморфизмами полупростых алгебраических групп", Докл. АН CCCP, 221:4 (1975), 767-770; англ. пер.: Е. В. Vinberg, "On the linear groups associated to periodic automorphisms of semisimple algebraic groups", Soviet Math. Dokl., 16 (1975), 406-409. 
[11] V.G. Kac, V.L. Popov, E. B. Vinberg, "Sur les groupes linéaires algébriques dont l'algèbre des invariants est libre", C. R. Acad. Sci. Paris Sér. A-B, 283:12 (1976), A $875-\mathrm{A} 878$.

[12] Э.Б. Винберг, "Группа Вейля градуированной алгебры Ли”, Изв. АН CССР. Сер. матем., 40:3 (1976), 488-526; англ. пер.: Еे. B. Vinberg, "The Weyl group of a graded Lie algebra", Math. USSR-Izv., 10:3 (1976), 463-495.

[13] V. Gatti [V. Kac], E. Viniberghi, "Spinors of 13-dimensional space", Adv. in Math., 30:2 (1978), 137-155.

[14] Э.Б. Винберг, А.Г. Элашвили, "Классификация тривекторов 9-мерного пространства", Тр. сем. по вект. и тенз. анализу, 18 (1978), 197-233; англ. пер.: È. B. Vinberg, A. G. Èlashvili, "Classification of trivectors of a 9-dimensional space", Selecta Math. Soviet., 7:1 (1988), 63-98.

[15] Э. Б. Винберг, Б. Н. Кимельфельд, "Однородные области на флаговых многообразиях и сферические подгруппы полупростых групп Ли", Функи. анализ и его прил., 12:3 (1978), 12-19; англ. пер.: É. B. Vinberg, B. N. Kimel'fel'd, "Homogeneous domains on flag manifolds and spherical subgroups of semisimple Lie groups", Funct. Anal. Appl., 12:3 (1978), 168-174.

[16] Э. Б. Винберг, "Классификация однородных нильпотентных элементов полупростой градуированной алгебры Ли", Тр. сем. по вект. и тенз. анализу, 19 (1979), 155-177; англ. пер.: Ѐ. В. Vinberg, "Classification of homogeneous nilpotent elements of a semisimple graded Lie algebra", Selecta Math. Soviet., 6 (1987), 15-35.

[17] Э. Б. Винберг, "Отсутствие кристаллографических групп отражений в пространствах Лобачевского большой размерности", Функи. анализ и его прил., 15:2 (1981), 67-68; англ. пер.: É. В. Vinberg, "Absence of crystallographic groups of reflections in Lobachevskii spaces of large dimension", Funct. Anal. Appl., 15:2 (1981), $128-130$.

[18] Э. Б. Винберг, "Отсутствие кристаллографических групп отражений в пространствах Лобачевского большой размерности", Тр. ММО, 47, Изд-во Моск. ун-та, M., 1984, 68-102; англ. пер.: Е. В. Vinberg, "The non-existence of crystallographic groups of reflections in Lobachevsky spaces of large dimension", Trans. Moscow Math. Soc., 1985, 75-112.

[19] È. B. Vinberg, "Discrete reflection groups in Lobachevsky spaces", Proceedings of the international congress of mathematicians, v. 1 (Warsaw, 1983), PWN, Warsaw, 1984, 593-601.

[20] Э.Б. Винберг, "Сложность действий редуктивных групп", Функи. анализ и его прил., 20:1 (1986), 1-13; англ. пер.: É. В. Vinberg, "Complexity of action of reductive groups", Funct. Anal. Appl., 20:1 (1986), 1-11.

[21] Э.Б. Винберг, А.Л. Онищик, Семинар по группам Ли и алгебраическим групnам, Наука, M., 1988, 344 с.; англ. пер.: A.L. Onishchik, E. B. Vinberg, Lie groups and algebraic groups, Springer Ser. Soviet Math., Springer-Verlag, Berlin, 1990, $\mathrm{xx}+328 \mathrm{pp}$.

[22] Э. Б. Винберг, А. Л. Онищик, "Основы теории групп Ли”, Группъ Ли и алгебры Ли - 1, Итоги науки и техн. Сер. Соврем. пробл. матем. Фундам. направления, 20, ВИНИТИ, М., 1988, 5-101; англ. пер.: А. L. Onishchik, Ѐ. B. Vinberg, "Foundations of Lie theory", Lie groups and Lie algebras I, Encyclopaedia Math. Sci., 20, Springer, Berlin, 1993, 1-94.

[23] Э. Б. Винберг, В. В. Горбацевич, О.В.Шварцман, “Дискретные подгруппы групп Ли", Группы Ли и алгебры Ли - 2, Итоги науки и техн. Сер. Соврем. пробл. матем. Фундам. направления, 21, ВИНИТИ, М., 1988, 5-120; англ. пер.: E. B. Vinberg, V.V. Gorbatsevich, O. V. Schwarzman, "Discrete subgroups of Lie groups", Lie groups and Lie algebras II, Encyclopaedia Math. Sci., 21, Springer, Berlin, 2000, 1-123. 
[24] Д. В. Алексеевский, Э. Б. Винберг, А. С. Солодовников, "Геометрия пространств постоянной кривизны", Геометрия - 2, Итоги науки и техн. Сер. Соврем. пробл. матем. Фундам. направления, 29, ВИНИТИ, М., 1988, 5-146; англ. пер.: D. V. Alekseevskij, E. B. Vinberg, A. S. Solodovnikov, "Geometry of spaces of constant curvature", Geometry II, Encyclopaedia Math. Sci., 29, Springer, Berlin, 1993, 1-138.

[25] Э. Б. Винберг, О.В.Шварцман, “Дискретные группы движений пространств постоянной кривизны", Геометрия - 2, Итоги науки и техн. Сер. Соврем. пробл. матем. Фундам. направления, 29, ВИНИТИ, М., 1988, 147-259; англ. пер.: E. B. Vinberg, O. V. Shvartsman, "Discrete groups of motions of spaces of constant curvature", Geometry II, Encyclopaedia Math. Sci., 29, Springer, Berlin, 1993, 139-248.

[26] Э. Б. Винберг, В. Л. Попов, “Теория инвариантов”, Алгебраическая геометрия - 4, Итоги науки и техн. Сер. Соврем. пробл. матем. Фундам. направления, 55, ВИНИТИ, М., 1989, 137-309; англ. пер.: V.L. Popov, Е. В. Vinberg, "Invariant theory", Algebraic geometry IV, Encyclopaedia Math. Sci., 55, Springer-Verlag, Berlin, 1994, 123-278.

[27] Э. Б. Винберг, В.В.Горбацевич, А. Л. Онищик, “Строение групп и алгебр Ли”, Группы Ли и алгебры Ли - 3, Итоги науки и техн. Сер. Соврем. пробл. матем. Фундам. направления, 41, ВИНИТИ, М., 1990, 5-253; англ. пер.: V.V. Gorbatsevich, A.L. Onishchik, E.B. Vinberg, "Structure of Lie groups and Lie algebras", Lie groups and Lie algebras III, Encyclopaedia Math. Sci., 41, Springer-Verlag, Berlin, 1994, 1-248.

[28] Э. Б. Винберг, "О некоторых коммутативных подалгебрах универсальной обертывающей алгебры”, Изв. АН СССР. Сер. матем., 54:1 (1990), 3-25; англ. пер.: 亡̀. В. Vinberg, "On certain commutative subalgebras of a universal enveloping algebra", Math. USSR-Izv., 36:1 (1991), 1-22.

[29] E. B. Vinberg, "On reductive algebraic semigroups", Lie groups and Lie algebras: E. B. Dynkin's seminar, Amer. Math. Soc. Transl. Ser. 2, 169, Adv. Math. Sci., 26, Amer. Math. Soc., Providence, RI, 1995, 145-182.

[30] Э. Б. Винберг, Курс алгебры, Факториал, М., 1999, 527 с.; англ. пер. 2-го изд.: E. B. Vinberg, A course in algebra, Grad. Stud. Math., 56, Amer. Math. Soc., Providence, RI, 2003, x+511 pp.

[31] D. N. Akhiezer, E. B. Vinberg, "Weakly symmetric spaces and spherical varieties", Transform. Groups, 4:1 (1999), 3-24.

[32] Э. Б. Винберг, "Коммутативные однородные пространства и коизотропные симплектические действия", УМH, 56:1(337) (2001), 3-62; англ. пер.: Е. В. Vinberg, "Commutative homogeneous spaces and co-isotropic symplectic actions", Russian Math. Surveys, 56:1 (2001), 1-60.

[33] Э. Б. Винберг, "Коммутативные однородные пространства гейзенбергова типа", Tp. MMO, 64, УРCC, М., 2003, 54-89; англ. пер.: Еे. В. Vinberg, "Commutative homogeneous spaces of Heisenberg type", Trans. Moscow Math. Soc., 2003 (2003), 45-78.

[34] E. B. Vinberg, "Some free algebras of automorphic forms on symmetric domains of type IV", Transform. Groups, 15:3 (2010), 701-741.

[35] Э.Б.Винберг, О.В.Шварцман, "Критерий гладкости в бесконечности арифметического фактора трубы будущего", Функи. анализ и его прил., 51:1 (2017), 40-59; англ. пер.: O. V. Shvartsman, E. В. Vinberg, "A criterion of smoothness at infinity for an arithmetic quotient of the future tube", Funct. Anal. Appl., 51:1 (2017), 32-47.

[36] Э.Б. Винберг, "О некоторых свободных алгебрах автоморфных форм", Функи. анализ и его прил., 52:4 (2018), 38-61; англ. пер.: Е. В. Vinberg, "On some free algebras of automorphic forms", Funct. Anal. Appl., 52:4 (2018), 270-289. 